\title{
Gallstone perforation of the ileum without obstruction
}

\author{
S. SHANKAR* \\ F.R.C.S.
}

\section{Summary}

A case of perforated ileum due to an impacted gall stone without associated intestinal obstruction, is reported. The problem in diagnosis and management are discussed.

KEY WORDS: perforated ileum, gallstones.

\section{Introduction}

Gallstone ileus is a well recognized complication of cholelithiasis, usually associated with one or other form of cholecysto-enteric or choledocho-enteric fistula (Day and Marks, 1975). No case of perforated ileum due to asymptomatic impacted gallstone is known by us to have been previously reported. The presentation and problems of management are described.

\section{Case report}

A 74-year-old woman was admitted for investigation of progressive anorexia and weight loss. She had been under medical treatment for congestive cardiac failure and hypothyroidism for some months. She admitted to post-prandial vomiting, but had no dysphagia. There was no history of dyspepsia or jaundice. Her exercise tolerance was markedly diminished.

On examination she was a frail old woman with evidence of recent weight loss. She was pale and had dry skin with senile purpura. She was not jaundiced. Her pulse was $104 / \mathrm{min}$ and irregular.

Barium meal revealed a fistula between the common hepatic duct and the first part of the duodenum. The duodenal cap appeared normal. There was no barium in the cystic duct and common bile duct, distal to the fistula. In addition, there was a large

*Present address: Wexham Park Hospital, Slough, Berks.

†Present address: St James' Hospital, Balham, London SW12.

\author{
J. W. P. BRADLEY \\ F.R.C.S.
}

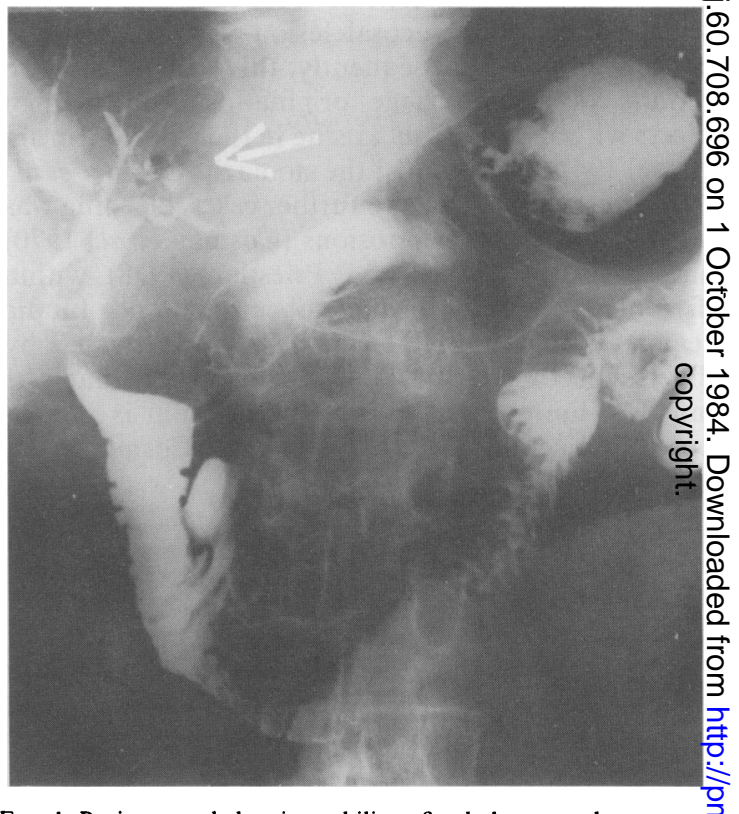

FIG. 1. Barium meal showing a biliary fistula between the commo bile duct and the first part of the duodenum. Air is present in the biliary tree (arrow).

duodenal diverticulum in the medial aspect of the second part of the duodenum (Fig. 1). In view of her poor condition, management was conservative.

A month later the patient developed acute colick yo abdominal pain. On examination, she was ill with at. blood pressure of $80 / 60 \mathrm{mmHg}$. Examination of the abdomen revealed generalized tenderness with marked guarding over the lower abdomen. BoweP sounds were absent.

A diagnosis of peritonitis due to a perforated viscus was thought the most likely cause, and despite the patient's poor general condition laparotomy was performed. This revealed a large volume of free fluid ${ }^{+}$ in the peritoneal cavity. Three faceted gallstones? 
were found in the terminal ileum $5 \mathrm{~cm}$ from the ileocaecal junction. One of the stones was associated with a $1-\mathrm{cm}$ perforation on the mesenteric border of the ileum (Fig. 2). The ileum was mildly distended proximal to the site of gallstone impaction.

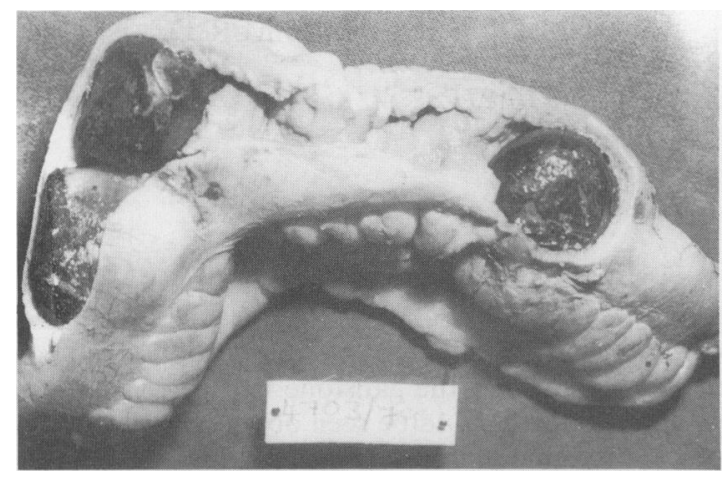

FIG. 2. Resected ileal segment containing three gallstones.

Examination of the biliary system confirmed the presence of cholecystoduodenal and choledochoduodenal fistulae. There was an accessory bile duct and a fibrotic gall bladder.

The affected ileal segment containing the gall stones was resected. Cholecystectomy was carried out after exploration of the common bile duct, which revealed a further stone. The fistulous tracts were excised and a choledochoduodenostomy was performed with T-tube drainage. The patient died of bronchopneumonia $48 \mathrm{hr}$ later.

\section{Discussion}

According to Railford (1962), gallstone ileus accounted for $2 \%$ of cases of intestinal obstruction, with the majority occurring in females $(F: M-7: 1)$. In patients aged 65 years and over, gallstones were the cause of intestinal obstruction in $25 \%$ of cases, with female preponderance (Day and Marks, 1975; Middleton and Muscroft, 1980). The commonest site of impaction of gallstones subsequent to their entry into the bowel is the terminal ileum (72).

Biliary fistulae are uncommon complications of cholelithiasis (0.3-0.5\%) (Day and Marks, 1975). Their presence is 3-16 times more common in women than men (Fox, 1970). Many are symptomless and are coincidentally discovered during routine investigations. Most commonly, the fistula extends between the gall bladder and the duodenum.

The case reported here is unusual in several ways. There was no clear history of biliary tract disease and the biliary fistula was revealed during barium meal performed as part of investigations of weight loss. It was assumed at this stage that any gallstones were probably small and would pass spontaneously per rectum. Surgery was not undertaken on account of her poor general condition. Subsequently, when the patient presented as an acute abdomen due to a probable perforation, laparotomy was performed, which revealed a perforated ileum due to a gallstone.

In retrospect, there are two aspects of management in this case which need critical evaluation. The first relates to the discovery of a symptomless biliary fistula in an elderly female who was unfit for surgery. The second is rather more controversial in that, when circumstances force a laparotomy, should the surgical procedure be confined to appropriate treatment of the affected ileal segment curtaining the gallstones followed by ileal anastomosis?

The initial conservative approach in this patient was dictated by her general condition although in different circumstances elective surgery might be justified to manage the biliary fistula. Cholecystectomy with T-tube drainage was carried out in this patient at the time of laparotomy to avoid a second operation, and also to prevent recurrence of gallstone impaction in the ileum. Ideally the biliary fistula would have to be dealt with at a later date. Cholecystectomy with closure of the fistula at primary operation has been recommended to prevent recurrence of gallstone ileus (Cooperman, Dickson and Remine, 1968).

We have been unable to find a previously reported case of spontaneous perforation of the ileum due to gallstones without associated clinical obstruction.

\section{Acknowledgments}

We are grateful to the Pathology Department at Mount Vernon Hospital for giving us permission to publish the photograph of the ideal segment with gallstones.

We also wish to thank Miss A. Kent and Mr D. Griffin for their valuable help in preparing the illustration.

\section{References}

Cooperman, A.M., Dickson, E.R. \& Remine, W.H. (1968) Changing concepts in the surgical treatment of gallstone ileus. Annals of Surgery, 167, 377.

DAY, A.E. \& MARKS, C. (1975) Gallstone ileus-review of the literature and presentation of thirty new cases. American Journal of Surgery, 104, 383.

Fox, P.F. (1970) Planning the operation for cholecysto-enteric fistula with gallstone ileus. Surgical Clinics of North America, 50, 93.

Middleton, M.D. \& MUSCROFT, J.J. (1980) Duodenal obstruction due to gallstones. Journal of the Royal College of Surgeons of Edinburgh, 25, 41.

RAILFORD, J.S. (1962) Intestinal obstruction caused by gallstones. American Journal of Surgery, 104, 383. 